\title{
Ergonomics as a tool in prolonging youth and postponing ageing
}

\author{
Ülo Kristjuhan \\ Department of Work Science and Safety, Tallinn University of Technology, Ehitajate tee 5, 18096 Tallinn, Estonia
}

\begin{abstract}
The EU and its Member States have identified population ageing as one of the key economic and social challenges to be faced. A high employment rate for older workers is essential. The ability to work is a prerequisite for a high employment rate for older workers. From 1965-2000, we carried out physiological and ergonomic studies in enterprises in Estonia, Russia, Ukraine and Moldova and proposed measures to avoid health risks. Our investigations were commissioned by industrial enterprises to improve the workplace and working conditions. The most interesting finding was that it was possible to markedly postpone the development of ageing changes in workers' bodies by up to 20 years without major investments.
\end{abstract}

Keywords: ergonomics, youth, ageing

\section{Introduction}

The EU and most other developed countries have identified population ageing as one of the key economic and social challenges to be faced. The policy of increasing employment among older workers is essential. Employment of older workers depends on many factors, including pension systems. However, the ability to work is a prerequisite for a high employment rate among older workers. The ability to work depends on the rate of accumulation of damage in workers' bodies during their lifetime. The different degree of accumulation of damage in people depends not only on the material substrate of the human body, but also on adverse environmental factors, including physical, chemical, psychological and social factors. An ergonomic approach can diminish the influence of adverse environmental factors.

\section{Concepts and definitions}

Vague concepts are poor tools for scientists. Therefore, fixed concepts and adequate definitions are essential preconditions to obtaining controlled information about the possibilities of postponing human ageing. Let us understand ageing as the accumulation of damage at the level of cells, organs, systems and the body during our lifetime that increases the possibility of death. According to the International
Ergonomics Association, ergonomics is the scientific discipline concerned with the understanding of interactions among humans and other elements in a human-machine-environment system. Ergonomics promotes a holistic approach in which considerations of physical, cognitive, social, organizational, environmental and other relevant factors are taken into account. Factually, the health effect of ergonomics interventions can be similar to health promotion.

\section{Advantages of ergonomic approach}

An ergonomic approach is effective in eliminating health risks for a number of reasons.

- It is mainly based on the systems approach. Very effective is macro-ergonomics - the ergonomic approach to big systems.

- Ergonomists use very different professional skills (engineering, psychology, health, toxicology etc.) to eliminate health risks.

- Ergonomics uses sensitive subjective methods (studies of sensation, discomfort and feelings) which enable the detection of weak physiological changes in different tissues of the human body earlier than most objective methods. It enables the development of pathological changes to be avoided.

- Ergonomics uses a participatory approach that respects the knowledge and experience of the user of ergonomic interventions. This approach enables the use of the wide vague knowledge of the user. 


\section{Contingents and methods}

From 1965-2000 we carried out physiological and ergonomic studies in enterprises in Estonia, Russia, Ukraine and Moldova and proposed ergonomic and health promotion measures. Our investigations were commissioned by industrial enterprises to improve the workplace and working conditions. The subjects of the studies (2147) were workers in different positions working in such industries as textiles, clothing, footwear, dairy automotive and building materials (garment workers, weavers, spinners, fitters, shoemakers, metal workers, engravers, sorters, smiths, bookkeepers etc.). Most of the groups studied included 30-50 male or female workers. We excluded workers who had health disorders. We used more than 30 different physiological and ergonomic methods that are able to be used under enterprise conditions: tests of the intensity of fatigue sensations and feelings, electromyography, a reaction time test, measurement of heart rate and critical flicker frequency, exact measurements of limb perimeters etc. The choice of methods was based on the aims of each study.

\section{Ergonomic methods developed by us}

We used different subjective methods of quantitative assessment of discomfort in industry and developed some new methods: special charts of 100 regions of the human body that took into account the qualitative and quantitative peculiarities of discomfort sensations. We also developed and used practical methods for precise measurement (to within $0.2 \mathrm{~mm}$ ) of the girth of human limbs for conditions of industry and services: perimeter gauges that consist of measuring tapes and loads and calibrated rulers. (The fluid volume in human lower limbs depends essentially on the body's position and on age.) The widespread use of these methods in industry and services has shown that they are effective in finding ways of preventing the accumulation of both pathological and non-pathological changes. We developed a special apparatus for the exact measurement (to within $0.1^{\circ}$ C) of skin temperature. We paid close attention to methods for studies of discomfort and its avoiding, as this is a warning sign of possible health disorders. On the basis of these data we provided recommendations to managers and at the individual level.

\section{Discomfort}

We paid a lot of attention to warning signals in the human body - primarily discomfort. We consider discomfort to be an antagonist to health that includes many sensations and unpleasant feelings (aversion to activity, being tired, itching, thinking about unpleasant things, anxiety, depression etc.). The human body 'speaks' to us all the time. There are many warning signals. Once you learn to listen to your body, you will discover a lot of information you need to fight illness and disease. Receptors in the body are highly sensitive to changes in tissues. These provide information to a subject about potential small adverse changes in tissues appearing earlier than changes detectable in laboratories. There are 'loud' signals and 'soft' signals. The need to end activities that cause pain in the region of the eyes or the need for sleep can be 'loud' signals from the body. There are also 'soft' signals, e.g. various feelings including discomfort, that our bodies give us. Researchers have studied them very little despite their importance in prevention. Our studies have shown that feelings of discomfort fulfil an important practical role as warnings of possible ageing-related health disorders [3]. In fact, people often ignore what their body is telling them and do not take any measures.

- They do not know about the possible risk factors.

- They do not have the possibility to eliminate this factor.

- $\quad$ People are so absorbed in their work that they do not even notice the signals.

- There are sometimes rare situations in which people pay too much attention to bodily sensations that disturb their health.

\section{Findings}

We recommended changing technology and work organization, corrective measures of ergonomics, self-care procedures or seeing a doctor, the right diet, preventive exercises to decrease discomfort and improving labour productivity. The most interesting finding was that it is possible to markedly postpone the development of age-related changes (including such age-related diseases as musculoskeletal disorders and cardiovascular diseases) in the body by up to 20 years without major investments. 


\section{Discussion}

We demonstrated possibilities of tackling the ageing population challenge: specific and effective measures designed to postpone ageing and increase the working ability of older workers. Also, we improved workers' health and thus saved on social expenses. Relatively high efficiency of proposed measures point to the theory of ageing caused by inadequate organism-environment interaction [1-2]. We did not find special works in scientific literature in which ergonomics was used to postpone ageing. However, some lifestyle studies have shown an essential effect. Longitudinal studies of 23,657 men [4] have shown that the risk of all-cause mortality was dramatically lower in the presence of just three 'lowrisk' factors (waist circumference of less than $94 \mathrm{~cm}$, physically active and did not smoke). Those who had these risk factors had a life expectancy which was 14 years shorter.

\section{References}

[1] A.V. Khalyavkin, The updated view concerning the possibility of growing old without senescence, Rejuvenation Res 12 (Suppl.1,2009), S-36.

[2] A.V. Khalyavkin \& A. Yashin, Non-pathological senescence arises from unsuitable external influences, Ann N Y Acad Sci, 1119 (2007) 306-309.

[3] Ü. Kristjuhan, Decreasing aging velocity in industry workers, Ann N Y Acad Sci, 1197 (2010) 49-53.

[4] C. D. Lee, X. Sui \& S. N. Blair, Combined effects of cardiorespiratory fitness, not smoking and normal waist girth on morbidity and mortality in men, Arch Intern Med 169 (2009) 2096-2101. 